\title{
ANALYSIS ON THE INHIBITION OF AGGREGATIBACTER ACTINOMYCETEMCOMITANS BY STREPTOCOCCUS SALIVARIUS ISOLATED FROM SALIVA AND TONGUE DORSUM OF ADULTS
}

\author{
REGINA VANIABELLA, HEDIJANTI JOENOES, BOY M BACHTIAR*
}

Department of Oral Biology, Faculty of Dentistry, Universitas Indonesia, Jakarta, Indonesia. Email: boybachtiar@gmail.com

Received: 21 April 2017, Revised and Accepted: 13 July 2017

\section{ABSTRACT}

Objective: To analyze the inhibitory potential of $S$. salivarius isolated from saliva and the dorsum of the tongue, and the protein it produces, in inhibiting the growth of Aggregatibacter actinomycetemcomitans.

Methods: Examine the inhibition zone of A. actinomycetemcomitans formed by the treatment of $S$. salivarius isolated from saliva and the tongue dorsum, along with the protein produced, in 10 participants using a deferred antagonism test with well-diffused gelatin.

Results: The inhibitory zone of $S$. salivarius isolated from saliva and the tongue against $A$. actinomycetemcomitans is insignificantly different ( $\mathrm{p} \geq 0.05$ ). There is no inhibition of the growth of A. actinomycetemcomitans shown by whole-cell and spent medium proteins of $S$. salivarius.

Conclusion: $S$. salivarius isolated from both saliva and the tongue dorsum is able to inhibit the growth of $A$. actinomycetemcomitans, but not the protein they produce.

Keywords: Streptococcus salivarius, Aggregatibacter actinomycetemcomitans, Lantibiotic, Probiotic.

(C) 2017 The Authors. Published by Innovare Academic Sciences Pvt Ltd. This is an open access article under the CC BY license (http://creativecommons. org/licenses/by/4. 0/) DOI: http://dx.doi.org/10.22159/ajpcr.2017.v10s5.23081

\section{INTRODUCTION}

Probiotics, according to the World Health Organization, are live microorganisms which when administered in adequate amounts are capable of providing health benefits for the recipient [1]. In recent years, research related to the use of oral probiotics for the prevention of dental caries and plaque biofilm formation, as well as for the treatment of halitosis and pharyngitis, has been successfully performed [1]. The aim of probiotic use in the oral cavity is to help prevent these diseases. Probiotics work by inhibiting the adhesion of pathogens in host tissues, stimulating and modulating the immune system of the mucosa, modulating apoptosis in cell proliferation, and improving the integrity of the barrier. Probiotics also kill or inhibit the growth of pathogens through the production of antimicrobial compounds such as bacteriocins or other products that are antagonistic against pathogens [2]. Unlike antibiotics, bacteriocins kill the bacteria within a relatively narrow spectrum [3]. Several studies have been conducted on the various multidrug-resistant pathogens that require natural antibiotic alternatives to restrict the use of antibiotic additives. One such alternative is the lantibiotic produced by Streptococcus salivarius.

S. salivarius is an essential element of biofilms in healthy individuals that colonize the oral mucosa, the dorsum of the tongue, and the pharyngeal mucosa [4]. S. salivarius is found as a pioneer colony in the human oral cavity from an early age. Its presence in the oral cavity can be identified within 2 days after birth and will settle as the predominant bacteria [1]. The number of $S$. salivarius in healthy adults can be as much as $2 \%$ from the isolated buccal mucosa, $17 \%$ from the tongue, and $30 \%$ from the pharynx. In the saliva samples of an adult population, S. salivarius' levels range from $10^{6}$ to $10^{7}$ colony-forming units (CFU)/ml [1]. S. salivarius has strains that are able to produce oral probiotics by releasing lantibiotics in large amounts in saliva, which in turn are able to eliminate harmful bacteria [4]. A lantibiotic is an antimicrobial peptide or small protein produced by the bacteria, which is able to kill or inhibit the growth of microorganisms. The lantibiotic produced by $S$. salivarius plays an important role in stabilizing the oral microbiota, preventing the excessive growth of pathogens, and preventing infections such as periodontitis.

In Indonesia, as in other developing countries, periodontal disease is an oral health problem for all age groups. Periodontal disease in Indonesia has the second highest prevalence after caries, which has an incident rate of $60 \%$ [5]. This high prevalence should encourage dentists to take a more proactive role with patients in preventing periodontitis in the coming years. Periodontitis is an infection of the supporting structures of the teeth caused by inflammation due to the formation of plaque and calculus from bacteria. This is followed by periodontal pocket formation which then results in progressive damage to the periodontal ligament and alveolar bone structure. The disease is stimulated by $90 \%$ of facultative anaerobic bacteria and $75 \%$ of which are Gramnegative bacteria. Some of the main causes of periodontal disease include inflammation of the periodontal tissues, attachment loss in the alveolar bone structure, and the accumulation of bacteria such as Aggregatibacter actinomycetemcomitans, which triggers the formation of microbial plaque.

A. actinomycetemcomitans is part of the normal flora in healthy individuals and is the predominant pathogen associated with localized aggressive periodontitis. A. actinomycetemcomitans has a variety of virulence factors, including the leukotoxin gene, which plays a role in the decline of the immune response and degradation of gingival epithelial attachment on periodontal tissues. Treatments given to patients with periodontitis may include conventional mechanical treatments such as scaling, root planing, and surgical intervention. However, patients with periodontitis caused by the bacterium A. actinomycetemcomitans often require not only conventional mechanical treatment but also antibiotic therapy. This is because the bacteria are able to invade the entire soft tissue and quickly colonize the periodontal pocket after doing mechanical therapy without antibiotics. Therefore, probiotic agents are expected to play a role in stopping or reducing the bacterial growth of A. actinomycetemcomitans in the oral cavity. Although the effects of $S$. salivarius probiotics are widely known, there has not been any research until now that proved the inhibitory potency of the lantibiotic released 
from S. salivarius against the growth of A. Aggregatibacter. Thus, this study is expected to show the presence/absence of antimicrobial activity of the lantibiotic produced by $S$. salivarius from the isolated saliva and tongue dorsum of healthy adult participants that affects the growth of A. actinomycetemcomitans.

\section{METHODS}

The procedure began with the selection and preparation of 10 participants. They were chosen in accordance with the inclusion criteria and given an explanation of the research to be conducted. They were each asked to sign an informed consent form if they understood and agreed to become a research participant. The participants were asked not to eat for $3 \mathrm{hrs}$ before sampling and to rinse with water to remove food debris. We then took samples of stimulated saliva and swabbed the dorsum of the tongue for each participant. Stimulated saliva sampling was conducted in the morning in the form of whole saliva up to $10 \mathrm{ml}$. Participants were instructed to chew Parafilm $\mathrm{M}{ }^{\circledR}$, and their saliva was collected in a sterile vial. Dorsum of the tongue swab sampling was also done in the morning using a Cytobrush from the circumvallate papillae to the tip of the tongue. Swabs collected on the Cytobrush were placed in a vial containing a solution of phosphatebuffered saline (PBS). The samples of saliva and tongue dorsum swabs that had been obtained were stored in the refrigerator (freezer) at $4^{\circ} \mathrm{C}$.

Next, we conducted the identification and confirmation of the $S$. salivarius colonies. Samples of saliva and tongue dorsum swabs were cultured on the selective medium mitis Salivarius agar (MSA) to isolate colonies of the $S$. salivarius strain. Afterward, colonies morphologically considered S. salivarius were confirmed by polymerase chain reaction (PCR), with S. salivarius ATCC 13419 as the positive control. PCR was performed in 30 cycles with a denaturation temperature of $94^{\circ} \mathrm{C}$ for 30 seconds, an annealing temperature of $55^{\circ} \mathrm{C}$ for 30 seconds, and an elongation time of 30 seconds at a temperature of $65^{\circ} \mathrm{C}$, using primers SalAUS (5'-GTAGAAAATATTTACTACATACT) and SalADS (5'GTTAAAGTA

TTCGTAAAACTGATG). PCR results were then input running on electrophoresis for 30 minutes $(100 \mathrm{~V})$ using a $1 \%$ agarose. They were stained with GelRed ${ }^{\mathrm{TM}}$, and then observed in the Gel Doc system. A DNA band of 118 base pairs indicated that the colonies identified on the medium MSA were $S$. salivarius. Next, the identification of the protein produced by $S$. salivarius through sodium dodecyl sulfate polyacrylamide gel electrophoresis (SDS-PAGE) started with the clinical culturing of S. salivarius colonies and ATCC 13419 in brain-heart infusion (BHI) broth for $18 \mathrm{hrs}$. Then, the pellets and spent medium were separated by centrifugation, and the pellets resuspended with a cell lysate buffer solution. These proteins were then analyzed using SDS-PAGE (150 V, $80 \mathrm{~mA}, 60$ minutes) and stained with Coomassie blue solution to see whether the S. salivarius proteins had the same molecular mass $(\mathrm{kDa})$. Samples with the same protein profile were then calculated its concentration with Bradford test to be used in the welldiffused gelatin methods.

Subsequently, the $S$. salivarius inhibitory potency test was performed against $A$. actinomycetemcomitans. Clinical S. salivarius and S. salivarius ATCC 13419 were diluted to 4 concentrations ( $\mathrm{CFU} / \mathrm{ml}$ ), then inoculated in a 1-cm line in the middle of the BHI gelatin medium and incubated for $24 \mathrm{hrs}$. Afterward, S. salivarius that grew on the surface was removed with the edge of the glass slide. The surface was sterilized with chloroform for 30 minutes then left for 15 minutes to remove the remnants of chloroform. We took one colony from A. actinomycetemcomitans and placed it in an Eppendorf tube which contained soft gelatin, in vortex, and removed it using a cotton bud. The cotton bud was then rubbed in a line perpendicular to the former $S$. salivarius swabs that had been cleaned. Each procedure was performed twice in duplicate. After that, colony was incubated for $24 \mathrm{hrs}$. Then, the growth inhibition zone of A. actinomycetemcomitans was measured by calculating the distance between the edges of the sweep of $S$. salivarius with a bacterial colonies growing test.
After the inter-bacterial inhibitory potency test, we tested the potential of the inhibitory protein S. salivarius against A. actinomycetemcomitans. A. actinomycetemcomitans was taken as many as 2 ose, put in an Eppendorf tube containing PBS, and in vortex until homogeneous. Then, $100 \mathrm{ml}$ of BHI gelatin was sterilized and cooled to $55^{\circ} \mathrm{C}$. The A. actinomycetemcomitans which had been dissolved in PBS was inoculated into the BHI gelatin that was still molten, put on an orbital shaker for 30 seconds, and poured into a Petri dish. When the gelatin had hardened, we made four wells of gelatin, each with a diameter of $4 \mathrm{~mm}$. The protein with four different concentrations $(\mathrm{pg} / \mathrm{ml})$ was inserted into each well and incubated for $24 \mathrm{hrs}$. We then observed and measured the distance between the edge of the well and the growth of the bacterial colonies. To process and analyze the data, we began with the Shapiro-Wilk data normality test. We then calculated the value of the average zone of inhibition and the protein produced by S. salivarius from both isolated sources to the growth of A. actinomycetemcomitans. We compared the results using the one-way ANOVA test and followed that with a post-hoc test. We compared the inhibitory potential of $S$. salivarius isolated from the saliva with the $S$. salivarius isolated from the tongue and its protein against $A$. actinomycetemcomitans using a paired t-test. Then, we used the Pearson's test to examine the correlation between the increase in concentration of $S$. salivarius and the protein with the inhibitory zone widening of A. actinomycetemcomitans.

\section{RESULTS}

The identification was done by comparing the morphology of S. salivarius from the culture of clinical samples with S. salivarius ATCC 13419 that was grown on the MSA medium. Twelve samples from six participants were tested using a PCR test to ensure that the colony was a colony of S. salivarius. Based on the PCR test results, we confirmed that all 12 colonies derived from the six participants were colonies of S. salivarius. Thus, it can be said that the results were not significant $(\mathrm{p} \geq 0.05)$ based on the identification of $S$. salivarius colonies on the mitis Salivarius gelatin medium and confirmed by PCR. Only 6 out of 10 participants showed evidence of S. salivarius in the oral cavity, as shown in Table 1.

Table 2 shows the results of the inhibitory potential of clinical S. salivarius isolated from both sources and S. salivarius ATCC 13419 against A. actinomycetemcomitans Y4 evaluated using a deferred antagonism test. However, of the four participants tested, only one participant's results could be interpreted. Consequently, for this part of the study, we only used two samples isolated from the saliva and tongue of a single participant.

The test results of both types of protein inhibitory potency against A. actinomycetemcomitans can be seen in Tables 3 and 4 . The amount of A. actinomycetemcomitans mixed in the gelatin medium in this method was $1.2 \times 10^{12} \mathrm{CFU} / \mathrm{ml}$.

Table 3 shows that there is no inhibition zone of A. actinomycetemcomitans that is generated by the whole-cell protein concentrations of $S$. salivarius isolated from either the saliva or the tongue. This is indicated by the colony of A. actinomycetemcomitans in the area around the well. The same can be seen in the test results of the protein inhibitory potential of the $S$. salivarius spent medium from both isolated sources against $A$. actinomycetemcomitans. Table 4 shows the absence of barriers that may occur from spent medium proteins. In addition, there were no obstacles against $A$. actinomycetemcomitans from the protein produced by S. salivarius ATCC 13419.

Table 1: Identification results of S. salivarius in clinical samples

\begin{tabular}{lll}
\hline Isolated source & \multicolumn{2}{l}{ S. salivarius colonies } \\
\cline { 2 - 3 } & Positive (\%) & Negative (\%) \\
\hline Saliva & $6(60)$ & $4(40)$ \\
Dorsum of the tongue & $6(60)$ & $4(40)$ \\
\hline
\end{tabular}

S. salivarius: Streptococcus salivarius 
Table 2: The result of the inhibitory potential of $S$. salivarius isolated from saliva and the dorsum of the tongue against A. actinomycetemcomitans

\begin{tabular}{|c|c|c|c|c|}
\hline \multirow{2}{*}{$\begin{array}{l}\text { Inhibitory potential } \\
\text { S. salivarius ATCC } 13419 \text { concentration (CFU/ml) }\end{array}$} & \multicolumn{4}{|c|}{ Culture results } \\
\hline & $1.7 \times 10^{7}$ & $1.7 \times 10^{8}$ & $1.7 \times 10^{9}$ & $1.7 \times 10^{10}$ \\
\hline Inhibitory zone & $0 \mathrm{~mm}$ & $0 \mathrm{~mm}$ & $0 \mathrm{~mm}$ & $0 \mathrm{~mm}$ \\
\hline Clinical S. salivarius strain concentration (saliva) (CFU/ml) & $4.4 \times 10^{10}$ & $4.4 \times 10^{9}$ & $4.4 \times 10^{8}$ & $4.4 \times 10^{7}$ \\
\hline Average of inhibitory zone & $3 \mathrm{~mm}$ & $3.5 \mathrm{~mm}$ & $6.5 \mathrm{~mm}$ & $9 \mathrm{~mm}$ \\
\hline Clinical S. salivarius strain concentration (tongue dorsum) (CFU/ml) & $3.7 \times 10^{10}$ & $3.7 \times 10^{9}$ & $3.7 \times 10^{8}$ & $3.7 \times 10^{7}$ \\
\hline Average of inhibitory zone & $3.25 \mathrm{~mm}$ & $6.75 \mathrm{~mm}$ & $6.5 \mathrm{~mm}$ & $7.75 \mathrm{~mm}$ \\
\hline
\end{tabular}

S. salivarius: Streptococcus salivarius, A. actinomycetemcomitans: Aggregatibacter actinomycetemcomitans, CFU: Colony-forming units

Table 3: The results of the inhibitory potency test of every whole-cell protein concentration of clinical S. salivarius and ATCC against A. actinomycetemcomitans

\begin{tabular}{|c|c|c|c|c|}
\hline \multirow{2}{*}{$\begin{array}{l}\text { Inhibitory potential } \\
\text { Whole-cell } S \text {. salivarius ATCC } 13419 \text { protein concentration }(\mu \mathrm{g} / \mathrm{ml})\end{array}$} & \multicolumn{4}{|c|}{ Culture results based on protein concentration } \\
\hline & $1.46 \times 10^{-2}$ & $1.46 \times 10^{-1}$ & 1.46 & 14.6 \\
\hline Inhibitory zone & $0 \mathrm{~mm}$ & $0 \mathrm{~mm}$ & $0 \mathrm{~mm}$ & $0 \mathrm{~mm}$ \\
\hline Whole-cell S. salivarius clinical strain protein concentration (saliva) $(\mu \mathrm{g} / \mathrm{ml})$ & $2.06 \times 10^{-2}$ & $2.06 \times 10^{-1}$ & 2.06 & 20.6 \\
\hline Inhibitory zone & $0 \mathrm{~mm}$ & $0 \mathrm{~mm}$ & $0 \mathrm{~mm}$ & $0 \mathrm{~mm}$ \\
\hline Whole-cell S. salivarius clinical strain protein concentration (tongue dorsum) $(\mu \mathrm{g} / \mathrm{ml})$ & $2.03 \times 10^{-2}$ & $2.03 \times 10^{-1}$ & 2.03 & 20.3 \\
\hline Inhibitory zone & $0 \mathrm{~mm}$ & $0 \mathrm{~mm}$ & $0 \mathrm{~mm}$ & $0 \mathrm{~mm}$ \\
\hline
\end{tabular}

S. salivarius: Streptococcus salivarius, A. actinomycetemcomitans: Aggregatibacter actinomycetemcomitans

Table 4: The results of the inhibitory potency test of every protein concentration of spent medium clinical S. salivarius and ATCC 13419 against A. actinomycetemcomitans

\begin{tabular}{|c|c|c|c|c|}
\hline \multirow{2}{*}{$\begin{array}{l}\text { Inhibitory potential } \\
\text { Spent medium } S \text {. salivarius ATCC } 13419 \text { protein concentration }(\mu \mathrm{g} / \mathrm{ml})\end{array}$} & \multicolumn{4}{|c|}{$\begin{array}{l}\text { Culture results based on protein } \\
\text { concentration }\end{array}$} \\
\hline & $1.81 \times 10^{-2}$ & $1.81 \times 10^{-1}$ & 1.81 & 18.1 \\
\hline Inhibitory zone & $0 \mathrm{~mm}$ & $0 \mathrm{~mm}$ & $0 \mathrm{~mm}$ & $0 \mathrm{~mm}$ \\
\hline Spent medium S. salivarius clinical strain protein concentration (saliva) $(\mu \mathrm{g} / \mathrm{ml})$ & $5.33 \times 10^{-2}$ & $5.33 \times 10^{-1}$ & 5.33 & 53.3 \\
\hline Inhibitory zone & $0 \mathrm{~mm}$ & $0 \mathrm{~mm}$ & $0 \mathrm{~mm}$ & $0 \mathrm{~mm}$ \\
\hline Spent medium $S$. salivarius clinical strain protein concentration (tongue dorsum) $(\mu \mathrm{g} / \mathrm{ml})$ & $2.79 \times 10^{-2}$ & $2.79 \times 10^{-1}$ & 2.79 & 27.9 \\
\hline Inhibitory zone & $0 \mathrm{~mm}$ & $0 \mathrm{~mm}$ & $0 \mathrm{~mm}$ & $0 \mathrm{~mm}$ \\
\hline
\end{tabular}

S. salivarius: Streptococcus salivarius, A. actinomycetemcomitans: Aggregatibacter actinomycetemcomitans

\section{DISCUSSION}

This study aimed to analyze the effectiveness of bacteria isolated from two sources: Saliva and the dorsum of the tongue, and the protein produced by $S$. salivarius, in inhibiting the growth of bacteria A. actinomycetemcomitans. S. salivarius colonies were identified by culture samples on the selective medium MSA and confirmed by PCR. We obtained data from 20 samples of isolated saliva and dorsal tongue swabs from 10 healthy adult participants and found that only $60 \%$ of the samples were positive for $S$. salivarius in the oral cavity $(\mathrm{p} \geq 0.05)$. This result differs from that of Wescombe et al., who found that $S$. salivarius is the pioneer colony in the oral cavity and will settle as the predominant bacteria on all human life [6].

The absence of S. salivarius in $40 \%$ of participants was also caused by the participants' dietary habits. In a previous study, it was demonstrated that the intake of sucrose is directly proportional to the number of Streptococcus salivarius on the tongue and saliva, which plays a role in the regulation of sucrose metabolism of this species $[7,8]$. One of the extracellular enzymes that are secreted by S. salivarius, fructosyltransferases, catalyzes the synthesis of sucrose into levan fructan with structures that are known to have a significant probiotic effect. In addition to dietary factors, enzymes in the saliva that have antibacterial properties such as lysozyme, lactoperoxidase, and amylase also affect the growth of S. salivarius in the oral cavity [9].

After identifying the colonies by PCR, we identified specific proteins produced by the colonies using SDS-PAGE. With this procedure, the whole-cell protein profiles (proteins that are inside the cell membrane) can be better identified than with the protein profile of the spent medium. This is consistent with research conducted by Barbour and
Philip (2014) which stated that $60-70 \%$ of bacteriocins discovered were peptides associated with producer cells of the cell wall, whereas $30-40 \%$ of the inhibitory peptide was secreted into the extracellular liquid medium. In addition, this study revealed that the production of lantibiotics by $S$. salivarius is regulated by a system of quorum sensing or interactions between cells, which occurs when the bacteria secrete peptide molecules that aid in bacterial communication. During the growth of bacteria, these peptides accumulate in the environment, and then, on the threshold of a certain concentration, trigger the production of lantibiotics in high concentrations. It can be concluded that the undetected proteins in the test results may have been caused by not achieving the threshold concentration through quorum sensing so that the production of lantibiotics was not triggered. The inhibitory potential of $S$. salivarius and the protein it produced against $A$. actinomycetemcomitans is distinguished based on the isolated source, the concentration of $S$. salivarius bacteria, and protein concentration. All culture procedures, bacteria inhibitory potency tests, and protein tests were done from the time of incubation for $24 \mathrm{hrs}$, with the goal of reaching the stationary growth phase of $S$. salivarius. In this phase, it produced secondary metabolites of $S$. salivarius, including the lantibiotic, and obtained stability of the inhibitory activity of the lantibiotic $[9,10]$.

In contrast to the Gram-positive bacteria, the Gram-negative bacteria have a cell wrapper structure consisting of three layers (trilayer): The outer membrane, the middle layer which is the cell wall, and the plasma membrane. According to Stevens et al., antibiotics have a bactericidal effect against Gram-positive bacteria by inducing the formation of porous bacterial cytoplasmic membranes and inhibiting the biosynthesis of the cell wall by binding with the molecular precursor cell walls and lipid II, 
which is located in the peptidoglycan [11]. Gram-negative bacteria such as A. actinomycetemcomitans are generally insensitive to lantibiotics, because the outer membrane of the Gram-negative bacteria which serves as a permeability barrier prevents the entry of macromolecules such as lantibiotic cells [9]. However, the vulnerability of cells to lantibiotics will increase when the outer membrane of the bacteria has been damaged or weakened by a permeabilizer.

A permeabilizer is an external agent capable of causing the loss of integrity of the outer membrane of the Gram-negative bacterial cell. Based on the research of Alakomi et al. (2000), the lactic acid produced by $S$. salivarius as a result of the fermentation of glucose is proven to work as a permeabilizer. Another study also stated that $S$. salivarius was able to ferment $93.6 \%$ of glucose in the medium and is the largest producer of lactic acid in the viridans group [12]. Therefore, the high concentration of lactic acid produced by $S$. salivarius may increase the sensitization of lantibiotics against $A$. actinomycetemcomitans. Alakomi et al. also stated that lactic acid as a permeabilizer is not bactericidal or bacteriostatic against Gram-negative bacteria. However, by increasing the permeability of the outer membrane, other compounds such as lantibiotics are able to penetrate and enhance the antimicrobial potency of these components against Gram-negative bacteria [10].

The interaction between the $S$. salivarius bacteria of isolated saliva and the dorsum of the tongue are one of the participants with A. actinomycetemcomitans bacteria which was tested with the deferred antagonism test. The test indicated that therewasnosignificantdifference between the inhibition zone of $A$. actinomycetemcomitans produced by $S$. salivarius isolated from saliva and the inhibition zone produced by the isolated samples from the tongue $(\mathrm{p} \geq 0.05)$. In the $S$. salivarius isolated saliva, the inhibition zone of A. actinomycetemcomitans was produced at a concentration of $10^{8} \mathrm{CFU} / \mathrm{ml}$ greater than the inhibition zone produced at a concentration of $10^{7} \mathrm{CFU} / \mathrm{ml}$, but not statistically significant. A significant difference was shown by the inhibition zone produced at a concentration of $10^{9}-10^{8} \mathrm{CFU} / \mathrm{ml}$, and $10^{10}$ with $10^{9} \mathrm{CFU} / \mathrm{ml}(\mathrm{p} \leq 0.05)$. Meanwhile, judging from the results of the inhibition zone of $A$. actinomycetemcomitans that was produced by $S$. salivarius isolated from the tongue, the only significant difference was found between the concentrations of $S$. salivarius $10^{7} \mathrm{CFU} / \mathrm{ml}$ with $10^{8} \mathrm{CFU} / \mathrm{ml}(\mathrm{p} \leq 0.05)$.

In addition, the isolated saliva showed an increase in the concentration of $S$. salivarius followed by an increase in the inhibition zone. Based on the results of the Pearson's correlation analysis, the concentration of $S$. salivarius from the isolated saliva showed a very strong positive correlation ( $\mathrm{p} \geq 0.05 ; \mathrm{r}=0887)$ with the resulting inhibition zone against A. actinomycetemcomitans. The same positive correlation was also demonstrated by $S$. salivarius isolated from the tongue, but only with a relatively strong correlation $(\mathrm{p} \geq 0.05 ; \mathrm{r}=0685)$. This proves that the inhibition zone of A. actinomycetemcomitans is affected by the concentration of $S$. salivarius. Based on the results of the paired t-test comparing the inhibition zone produced by $S$. salivarius from both isolated sources with the inhibition zone produced by $S$. salivarius ATCC as a positive control, the inhibitory potential of $S$. salivarius from both the saliva and the dorsum of the tongue (clinical strain) against A. actinomycetemcomitans was significantly better than $S$. salivarius ATCC (laboratory strains) $(p \leq 0.05)$. This is consistent with the research of a previous study, which stated that the results of the SDSPAGE mutant strain or laboratory strain were weaker than the clinical strain or wild-type [13]. Weerkamp and McBride also stated that the wild-type strain has adhesive properties that allow attachment to the buccal surface, teeth, and dorsum of the tongue, as well as aggregate with other bacteria, whereas $S$. salivarius ATCC laboratory strains do not adhere to the epithelial cells [14].

Furthermore, to analyze the antimicrobial activity exhibited by $S$. salivarius against A. actinomycetemcomitans in the deferred antagonism test, we tested the interaction of a protein produced by S. salivarius using well-diffused gelatin. In contrast to the results of the interaction between bacteria in the previous method, the protein interaction of $S$. salivarius with A. actinomycetemcomitans did not show the formation of barriers, either by whole-cell protein and spent medium, or from isolated saliva and tongue samples. The test results were similar with the $S$. salivarius ATCC protein. In accordance with the previous explanation of the bactericidal mechanism of lantibiotics against Gram-negative bacteria, the lantibiotic produced by $S$. salivarius showed antimicrobial action after the integrity of the outer membrane of bacteria was disturbed. Due to the relatively large size of the lantibiotic molecule (1800-4600 Da), it could not penetrate the outer membrane of Gram-negative bacteria [15]. In this case, it needed a permeabilizer such as the lactic acid produced by S. salivarius to penetrate the outer membrane [12]. In this method, exposure of whole-cell proteins and the spent medium was only done to test the bacteria, so the glucose fermentation process did not occur. Therefore, the lactic acid that was supposed to act as an agent permeabilizer to potentially disintegrate the outer membrane of A. actinomycetemcomitans was not formed, and the lantibiotic was not able to penetrate the cytoplasmic membrane of bacteria. This resulted in A. actinomycetemcomitans becoming insensitive to the lantibiotic $[9,10]$.

Furthermore, the previous study stated that a greater number of lantibiotics would be produced by S. salivarius if they were grown in a solid medium, whereas lantibiotics produced by $S$. salivarius cultured in a liquid medium would show a very weak expression of inhibitory activity [16]. According to their study, a liquid medium can be used if the auto-induction method is used to obtain bacterial proteins. The protein interactions test of $S$. salivarius against $A$. actinomycetemcomitans was derived from cultures of S. salivarius in a broth medium (liquid medium). Therefore, the protein produced by both the clinical $S$. salivarius and S. salivarius ATCC had a very weak antimicrobial action. In this study, we did not do a protein purification procedure or autoinduction to get a specific $S$. salivarius lantibiotic, due to available time and materials. In addition, the working mechanism of the lantibiotic produced by S. salivarius isolated from saliva and the dorsum of the tongue against A. actinomycetemcomitans cannot be explained further. Therefore, additional research is required to study the interaction of specific lantibiotic clinical strains of $S$. salivarius from isolated saliva and the dorsum of the tongue against $A$. actinomycetemcomitans.

\section{CONCLUSION}

We concluded that $S$. salivarius has an inhibitory potency against A. actinomycetemcomitans, but there is no difference in the potential inhibitory effect between $S$. salivarius isolated from saliva or the dorsum of the tongue. In addition, there are no barriers to the growth of A. actinomycetemcomitans by the protein produced by $S$. salivarius isolated from saliva and the tongue.

\section{REFERENCES}

1. Horz HP, Meinelt A, Houben B, Conrads G. Distribution and persistence of probiotic Streptococcus salivarius K12 in the human oral cavity as determined by real-time quantitative polymerase chain reaction. Oral Microbiol Immunol 2007;22(2):126-30.

2. Haukioja A. Probiotics and oral health. Eur J Dent 2010;4(3):348-55.

3. Fauzi AA, Shafiei Z, Baharin B, Mohd N. Isolation of Lactobacillus from periodontally healthy subjects and its antimicrobial activity against periodontal pathogens. Sains Malaysiana 2013;42(1):19-24.

4. Burton JP, Wescombe PA, Macklaim JM, Chai MH, Macdonald K, Hale JD, et al. Persistence of the oral probiotic Streptococcus salivarius M18 is dose dependent and megaplasmid transfer can augment their bacteriocin production and adhesion characteristics. PLoS One 2013;8(6):e65991.

5. Kementerian Kesehatan Republik Indonesia. Profil Kesehatan Indonesia Tahun 2011. Jakarta: Kementerian Kesehatan; 2012.

6. Wescombe PA, Hale JD, Heng NC, Tagg JR. Developing oral probiotics from Streptococcus salivarius. Future Microbiol 2012;7(12):1355-71.

7. Forssten SD, Björklund M, Ouwehand AC. Streptococcus mutans, caries and simulation models. Nutrients 2010;2(3):290-8

8. Roger P, Delettre J, Bouix M, Béal C. Characterization of Streptococcus salivarius growth and maintenance in artificial saliva. J Appl Microbiol 
2011;111(3):631-41.

9. McAuliffe O, Ross RP, Hill C. Lantibiotics: Structure, biosynthesis and mode of action. FEMS Microbiol Rev 2001;25(3):285-308.

10. Alakomi HL, Skyttä E, Saarela M, Mattila-Sandholm T, LatvaKala K, Helander IM. Lactic acid permeabilizes Gram-negative bacteria by disrupting the outer membrane. Appl Environ Microbiol 2000;66(5):2001-5.

11. Parada JL, Caron CR, Medeiros AB, Soccol CR. Bacteriocins from lactic acid Bacteria: Purification, properties and use as biopreservatives. Braz Arch Biol Technol 2007;50:521-42.

12. Burton JP, Drummond BK, Chilcott CN, Tagg JR, Thomson WM, Hale JD, et al. Influence of the probiotic Streptococcus salivarius strain M18 on indices of dental health in children: A randomized doubleblind, placebo-controlled trial. J Med Microbiol 2013;62:875-84.

13. Gauthier M, Brochu D, Eltis LD, Thomas S, Vadeboncoeur C.
Replacement of isoleucine-47 by threonine in the HPr protein of Streptococcus salivarius abrogates the preferential metabolism of glucose and fructose over lactose ana melibose but does not prevent the phosphorylation of $\mathrm{Hpr}$ on serine-46. Mol Microbiol 1997;25(4):695-705.

14. Weerkamp AH, McBride BC. Adherence of Streptococcus salivarius HB and HB-7 to oral surfaces and saliva-coated hydroxyapatite. Infect Immun 1980;30(1):150-8.

15. Brötz H, Sahl HG. New insights into the mechanism of action of lantibiotics-diverse biological effects by binding to the same molecular target. J Antimicrob Chemother 2000;46(1):1-6.

16. Barbour A, Philip K, Muniandy S. Enhanced production, purification, characterizatio $\mathrm{n}$ and mechanism of action of salivaricin 9 lantibiotic produced by Streptococcus salivarius NU10. PLoS One 2013;8(10):e77751. 